\title{
Evaluating the malignant potential of intraductal papillary mucinous neoplasms of the pancreas: added value of non-enhanced endoscopic ultrasound to supplement non-enhanced magnetic resonance imaging
}

\author{
Takao Igarashi ${ }^{\mathrm{A}, \mathrm{B}, \mathrm{C}, \mathrm{E}, \mathrm{F}}$, Hirokazu Ashida ${ }^{\mathrm{D}}$, Kazuhiko Morikawa ${ }^{\mathrm{D}}$, Keitaro Enoki ${ }^{\mathrm{D}}$, Kazuyoshi Ohki ${ }^{\mathrm{E}}$, Gou KawakamiE, \\ Hiroya Ojiri ${ }^{\mathrm{E}}$
}

Department of Radiology, The Jikei University School of Medicine, Tokyo, Japan

\section{Abstract}

Purpose: To evaluate the diagnostic performance of combining non-enhanced magnetic resonance imaging (MRI) and non-enhanced endoscopic ultrasonography (EUS) for assessing the malignant potential of lesions in patients with intraductal papillary mucinous neoplasms of the pancreas (IPMNs).

Material and methods: Data from 38 patients histopathologically diagnosed with IPMN adenomas or IPMN adenocarcinomas were retrospectively analysed. Preliminary univariate and multivariate analyses were conducted to identify statistically significant associations. Three blinded radiologists evaluated the image sets to assess the diagnostic performance of combined use of non-enhanced MRI and EUS as opposed to non-enhanced MRI alone in distinguishing malignant from benign lesions. Observer performance and interobserver variability were determined using receiver-operating-characteristic curve analysis and weighted $\kappa$ statistics.

Results: Multivariate analyses identified a significant difference between the abrupt change in the main pancreatic duct (MPD) calibre with distal pancreatic atrophy and the signal intensity of lesion-to-spinal cord ratio on MRI; a significant difference was observed in MPD size on EUS. Diagnostic performance assessments of the image sets did not differ significantly between the blinded radiologists.

Conclusions: The clinical utility of non-enhanced EUS may be attributive in evaluating IPMN that has already been evaluated by non-enhanced MRI.

Key words: magnetic resonance imaging, endoscopic ultrasonography, intraductal papillary mucinous neoplasm of the pancreas.

\section{Introduction}

Most cystic lesions of the pancreas are incidentally detected during ultrasonography, computed tomography (CT), or magnetic resonance imaging (MRI) combined with MR cholangiopancreatography (MRCP) performed because of an unrelated indication. Intraductal papillary mucinous neoplasm of the pancreas (IPMN) is a fairly common cystic lesion of the pancreas, particularly observed in the elderly. IPMN was first recognised in the 1980s; the volume of published literature related to IPMN has increased over the last decade alongside a sharp increase in incidence because of increased detection of such lesions. Asymptomatic cysts, most of which were presumably small IPMNs, were identified in $2.8 \%$ of 2832 consecutive CT scans performed in one year at a single institution [1]. IPMNs are defined as grossly visible intraductal epithelial neoplasms with papillary proliferation and cyst formation [2]. The 2010 World Health Organisation (WHO) classification subcategorises the IPMNs according to their malignant transformation as IPMN with low or intermediate dyspla-

Correspondence address:

Takao Igarashi, Department of Radiology, The Jikei University School of Medicine, 3-25-8, Nishi-Shimbashi, Minato-ku, Tokyo 105-8461, Japan,

phone: +81-3-3433-1111, fax: +81-3-3431-1775, e-mail: igarashi-†@jikei.ac.jp

Authors' contribution:

A Study design - B Data collection · C Statistical analysis · D Data interpretation - E Manuscript preparation · F Literature search · G Funds collection 
sia, IPMN with high-grade dysplasia (equivalent to the intraductal papillary mucinous carcinoma [IPMC], non-invasive), and IPMN with an associated invasive carcinoma (IPMC). In addition to pancreatic intraepithelial neoplasia (PanIN), the most common neoplastic precursor to ductal pancreatic cancer is IPMN [2,3]. Its relatively slow growth enables early diagnosis and curative surgical treatment [4]. IPMC has been attracting attention as a potentially curable pancreatic cancer if treated prior to becoming invasive.

Diffusion-weighted imaging (DWI) has been shown to be effective in the diagnosis of malignant IPMN [5-7]. Malignant pancreatic tumours generally show higher signal intensities and lower apparent diffusion coefficients (ADCs) than benign tumors [8]. The diagnostic performance of DWI was shown to be comparable to that of contrast-enhanced MRI for evaluating the malignant potential of cystic pancreatic lesions [7,9]. Furthermore, the combined use of DWI and MRCP with non-enhanced MRI was shown to be particularly effective in the diagnosis of malignant IPMN and prediction of invasive IPMC risk [5,7].

Endoscopic ultrasonography (EUS) is the best modality for monitoring pancreatic cystic neoplasms and for predicting the malignant potential of branch-type IPMN through the detection and evaluation of mural nodules [10,11]. The diameter and width of the mural nodules of papillary protrusions as assessed on preoperative EUS were shown to reliably distinguish between low- and high-risk IPMNs [12].

In situations in which there is an increased detection of IPMNs due to widespread use of imaging examinations, differentiating benign from potentially malignant ones using a noninvasive technique is of great value. In the screening test, the administration of contrast material is not necessary. For evaluating the malignant potential of IPMN using non-enhanced MRI combined with MRCP and DWI, the advantage of using non-enhanced EUS to supplement non-enhanced MRI is not clear. The minimal interobserver variability of assessing mural nodules or solid tumours would be invaluable for the administration of appropriate treatment in patients with suspicious lesions [5]. To our knowledge, the added value of non-enhanced EUS to the interpretation of findings from non-enhanced MRI combined with MRCP and DWI has not been investigated to date.

The purpose of our study was to evaluate the diagnostic value of combined use of non-enhanced MRI with MRCP and DWI and non-enhanced EUS (hereafter referred to as MRI with EUS), as opposed to non-enhanced MRI with MRCP and DWI alone (hereafter referred to as MRI alone).

\section{Material and methods}

\section{Patients}

The requirement for written, informed consent from patients was waived because of the retrospective nature of the review of clinical and imaging data. Patients who were suspected to have IPMN with malignant potential upon preoperative MRI and/or EUS and who underwent curative surgery for IPMN at our institution between June 01, 2009 and October 31, 2015 were retrospectively identified by searching our imaging database. Preoperative EUS with fine needle aspiration had never been performed in our institution. The following exclusion criteria were used: (a) patients with pancreatic ductal adenocarcinoma concomitant with IPMNs, characterised by distinct cystic lesions on histopathology; (b) patients with mucinous carcinoma on histopathology; (c) patients who did not undergo MRI or EUS; and (d) patients who did not undergo preoperative MRI and EUS within the immediate six months before the date of surgery. A detailed process of the patient selection is summarised in Figure 1.
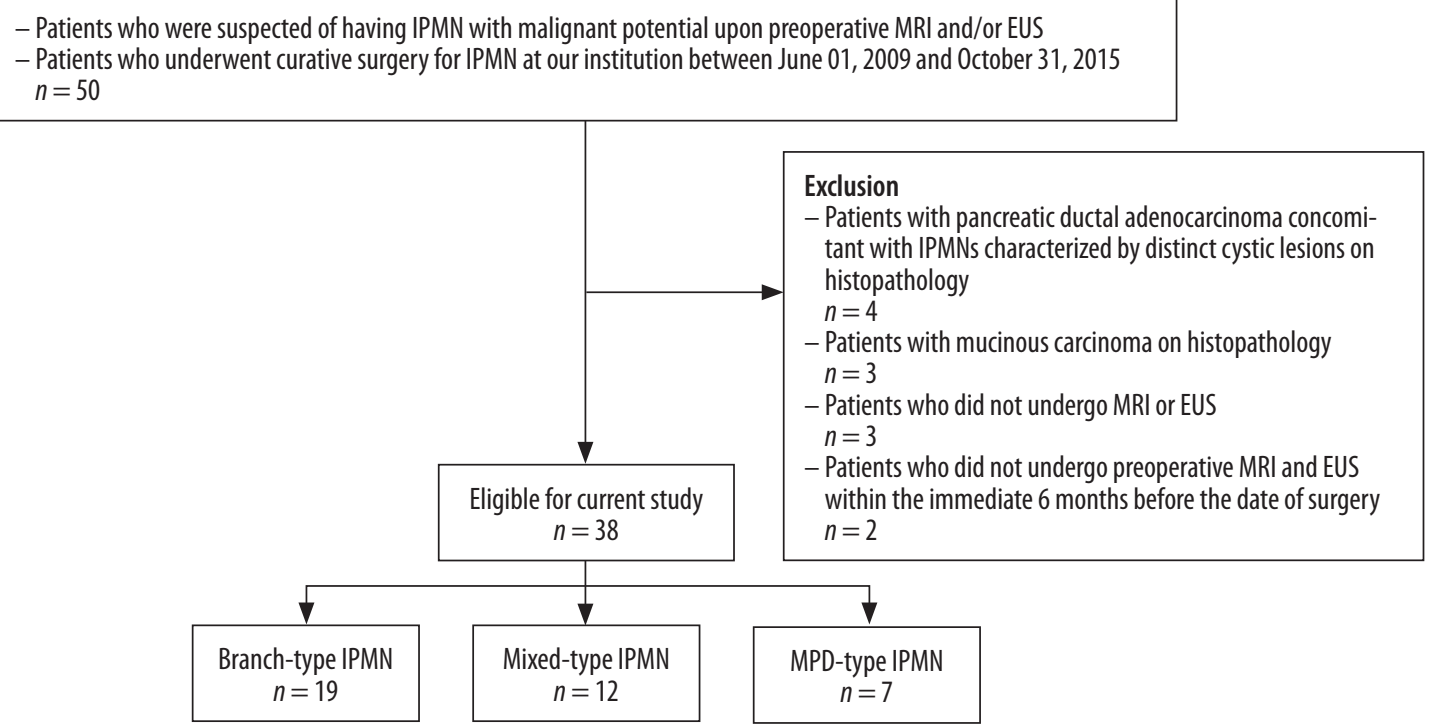

IPMN - intraductal papillary mucinous neoplasms, MRI - magnetic resonance imaging, EUS - endoscopic ultrasonography, MPD - main pancreatic duct

Figure 1. Flow diagram shows the process for patient selection 


\section{Magnetic resonance imaging technique}

All patients had undergone MRI on a 1.5 Tesla unit (MAGNETOM Avanto or Symphony, Siemens Medical Solutions, Erlangen, Germany) using a phased-array body coil. Non-enhanced fat-suppressed in-phase T1-weighted breath-hold spoiled gradient-recalled-echo (GRE) images, T2-weighted fast-spin-echo (T2W-FSE) images, DW images with low and high b-values $\left(b=50,800 \mathrm{~s} / \mathrm{mm}^{2}\right.$, respectively), and 3D-fat-suppressed T1-weighted GRE sequences with Cartesian sampling, named volumetric interpolated breath-hold examination (VIBE), on Siemens Healthcare MRI systems were obtained in the axial plane. Half-Fourier acquisition single-shot turbo spin-echo (HASTE) sequence was obtained in the axial and coronal planes. A respiratory-triggered 3D-T2-weighted MRCP sequence was acquired, and coronal maximum intensity projection (MIP) was reconstructed based on a dataset. We have included DWI as a routine protocol for MRCP conducted at our institution. Details of imaging parameters evaluated in this study are summarised in Supplementary Table 1.

\section{Endoscopic ultrasonography technique}

After obtaining written, informed consent, patients were sedated with midazolam using an intravenous bolus dose of 4-5 mg under haemodynamic and oximetric monitoring. EUS was performed by an endosonographer with 20 years of experience at our institution. An electronic radial (GF-UE260, Olympus Medical Systems, Tokyo, Japan) or a curved linear array echoendoscope (GF-UCT260, Olympus Medical Systems, Tokyo, Japan) with the ProSound Alpha-10 processor (Hitachi-Aloka Medical Co., Ltd., Tokyo, Japan) was used for endoscopic diagnosis. Tissue harmonic EUS screening of the pancreaticobiliary system was performed using standardised imaging techniques $[13,14]$; particular attention was paid to primary pancreatic lesions. Harmonic imaging was achieved with a transmitting frequency of $3.75 \mathrm{MHz}$ and receiving frequency of $7.5 \mathrm{MHz}$. The acoustic power of harmonic EUS was set with a mechanical index of $0.25-0.3$, and a single focus point was set at the most distant margin of the pancreatic lesions from the transducer. During EUS examination, tumour locations were recorded (head, body, and tail or diffuse). The cyst sizes and the thicknesses of cyst wall and/ or septum and mural nodules were measured. The associated presence of main pancreatic duct dilatation and/or an invasive cystic lesion component was recorded, if identified.

\section{Image analysis}

\section{Evaluation of imaging findings for preliminary analyses}

One radiologist with 15 years of MRI experience retrospectively reviewed the patient medical records at our in- stitution. The same radiologist, who was aware of the clinical information, evaluated both the interpreted $M R$ and EUS findings on a picture archiving and communication systems (PACS) monitor. According to the 2012 international consensus/Fukuoka guidelines (Fukuoka guidelines [FG]) for the management of IPMN and mucinous cystic neoplasms of the pancreas, which focus on high-risk stigmata (HRS) and worrisome imaging features (WFs) [10] the following morphological findings were assessed by the same radiologist: main pancreatic duct (MPD) size (> $10 \mathrm{~mm}$ or $5-9 \mathrm{~mm}$ ); cyst size (> $30 \mathrm{~mm}$ or not); wall and/or septal thickness of cyst (>3 mm or not); mural nodule height and/or width (> $10 \mathrm{~mm}, 5-9 \mathrm{~mm}$ or less); abrupt change in the MPD calibre with distal pancreatic atrophy (present or absent); and lymphadenopathy (present or absent). In addition to performing an assessment according to the FG criteria, the quantitative assessment of $\mathrm{ADC}$ value based on the ADC map, and the calculation of lesion-to-spinal cord ratio (LSR) as a relative ADC value were performed. Optimal cut-off values of ADC and LSR were identified on receiver-operating-characteristic (ROC) curve analysis. These optimal cut-off values of ADC and LSR were applied for blinded evaluation.

An electronic calliper included in the PACS program was used for the measurement of the largest diameter of lesions in any axial or coronal image. For cyst size and wall and/or septal thickness of cyst, the parameters of evaluation were branch-type and mixed-type IPMN. If several cysts were present, the cyst with the largest wall and/or septal thickness was accepted as a lesion. A mural nodule was defined as any solid nodule in the MPD or branch duct of a well-circumscribed tissue lesion surrounded by a duct wall. For lymphadenopathy, a diameter $>10 \mathrm{~mm}$ in the short axis was accepted as significant lymphadenopathy.

\section{Measurement of apparent diffusion coefficient and lesion- to-spinal cord ratio on diffusion-weighted images}

For the visual assessment of DW images, the ADC measurements were performed on ADC maps included in the PACS program. The region of interest (ROI) for obtaining the ADC value was manually selected. In the case of a solid component such as a mural nodule, thickened septa and/or cyst wall, or parenchymal mass, the ROI was selected to cover both the solid and the cystic portions as much as possible. For precise measurement of ADC values, the ROI was placed on the lesion of the HASTE image and was copied manually to the DW image to exclude a partial volume phenomenon. The mean ADC value calculated from at least three measurements was used in the analysis. T2W-FSE images were not used to determine a ROI because of their lower spatial resolution compared with that of HASTE images.

LSR was measured on the DW image with a diffusion gradient of $b=800 \mathrm{~s} / \mathrm{mm}^{2}$. For measurement of spinal cord signal intensity on DWI, the ROI was located on 
Table 1. Imaging findings in patients with intraductal papillary mucinous carcinomas (IPMC) and intraductal papillary mucinous adenomas (IPMAs)

A) MRI

\begin{tabular}{|c|c|c|c|c|c|c|}
\hline \multirow{2}{*}{ Factor } & \multirow{2}{*}{$\begin{array}{c}\text { IPMC } \\
(n=20)\end{array}$} & \multirow{2}{*}{$\begin{array}{c}\text { IPMA } \\
(n=18)\end{array}$} & \multirow{2}{*}{$\begin{array}{c}\text { Univariate } \\
p \text { value }\end{array}$} & \multicolumn{3}{|c|}{ Multivariate } \\
\hline & & & & $\mathrm{OR}$ & $95 \% \mathrm{Cl}$ & $p$ value \\
\hline Age, mean (SD) & $73.9(8.3)$ & $68.1(9.7)$ & 0.6 & - & - & - \\
\hline Sex (male, female) & 16,4 & 12,6 & 0.23 & - & - & - \\
\hline IPMN type & - & - & $<0.001$ & - & - & - \\
\hline Branch & 4 & 15 & - & - & - & - \\
\hline Mixed & 9 & 3 & - & - & - & - \\
\hline MPD & 7 & 0 & - & - & - & - \\
\hline Location & - & - & 0.36 & - & - & - \\
\hline Head & 14 & 10 & - & - & - & - \\
\hline Body & 5 & 4 & - & - & - & - \\
\hline Tail & 1 & 4 & - & - & - & - \\
\hline MPD size $(\mathrm{mm})$ & - & - & - & 5.02 & $0.61-41.4$ & 0.13 \\
\hline$\geq 10$ & 8 & 0 & 0.004 & - & - & - \\
\hline$\geq 5$ & 17 & 5 & $<0.001$ & - & - & - \\
\hline Cyst size (mm) & - & - & 0.45 & - & - & - \\
\hline$\geq 30$ & 7 & 13 & - & - & - & - \\
\hline$<30$ & 6 & 5 & - & - & - & - \\
\hline Wall and/or septal thickness of cyst (mm) & - & - & 0.1 & - & - & - \\
\hline$\geq 3$ & 6 & 3 & - & - & - & - \\
\hline$<3$ & 6 & 15 & - & - & - & - \\
\hline \multicolumn{7}{|l|}{ Mural nodule height and/or width (mm) } \\
\hline$\geq 10$ & 3 & 1 & 0.35 & - & - & - \\
\hline$\geq 5$ & 11 & 7 & 0.32 & - & - & - \\
\hline Abrupt change in MPD calibre with distal pancreatic atrophy & - & - & $<0.001$ & 34.5 & $1.85-644$ & 0.018 \\
\hline Present & 13 & 1 & - & - & - & - \\
\hline Absent & 7 & 17 & - & - & - & - \\
\hline Lymphadenopathy & - & - & 0.99 & - & - & - \\
\hline Present & 1 & 0 & - & - & - & - \\
\hline Absent & 19 & 18 & - & - & - & - \\
\hline ADC value $\left(\times 10^{-3} \mathrm{~mm}^{2} / \mathrm{s}\right)$ & $1.79( \pm 0.45)$ & $2.43( \pm 0.33)$ & $<0.001$ & - & - & - \\
\hline Area of ROI for ADC (mm²) & $734( \pm 693)$ & $798( \pm 793)$ & 0.62 & - & - & - \\
\hline LSR & $0.26( \pm 0.09)$ & $0.17( \pm 0.04)$ & $<0.001$ & 16.3 & $1.45-184$ & 0.02 \\
\hline Area of ROI for LSR & $91.8( \pm 12.1)$ & $84.5( \pm 11.4)$ & 0.08 & - & - & - \\
\hline
\end{tabular}

B) EUS

\begin{tabular}{|c|c|c|c|c|c|c|}
\hline & \multirow{2}{*}{$\begin{array}{c}\text { IPMC } \\
(n=20)\end{array}$} & \multirow{2}{*}{$\begin{array}{c}\text { IPMA } \\
(n=18)\end{array}$} & Univariate & \multicolumn{3}{|c|}{ Multivariate } \\
\hline & & & $p$ value & OR & $95 \% \mathrm{Cl}$ & $p$ value \\
\hline MPD size (mm) & - & - & $<0.001$ & 8.15 & $1.04-63.9$ & 0.045 \\
\hline$\geq 10$ & 9 & 0 & - & - & - & - \\
\hline$\geq 5$ & 15 & 4 & - & - & - & - \\
\hline Cyst size (mm) & - & - & 0.27 & - & - & - \\
\hline$\geq 30$ & 5 & 11 & - & - & - & - \\
\hline$<30$ & 8 & 6 & - & - & - & - \\
\hline
\end{tabular}


Table 1. Cont.

\begin{tabular}{|l|c|c|c|c|c|c|} 
& IPMC & IPMA & Univariate & \multicolumn{3}{c|}{ Multivariate } \\
\cline { 4 - 7 } & $(n=20)$ & $(n=18)$ & $p$ value & OR & $95 \%$ Cl & $p$ value \\
\hline Wall and/or septal thickness of cyst $(\mathrm{mm})$ & - & - & 0.02 & 0.7 & $0.07-6.74$ & 0.76 \\
\hline$\geq 3$ & 8 & 4 & - & - & - & - \\
\hline$<3$ & 3 & 14 & - & - & - & - \\
\hline Mural nodule height and/or width (mm) & - & - & 0.02 & 15.7 & $0.58-428$ & 0.102 \\
\hline$\geq 10$ & 8 & 1 & - & - & - & - \\
\hline$\geq 5$ & 13 & 7 & - & - & - & - \\
\hline Abrupt change in the MPD calibre with distal pancreatic atrophy & - & - & 0.001 & 6.43 & $0.53-77.8$ & 0.14 \\
\hline Present & 11 & 1 & - & - & - & - \\
\hline Absent & 9 & 17 & - & - & - & - \\
\hline Lymphadenopathy & - & - & 0.99 & - & - & - \\
\hline Present & 0 & 0 & - & - & - & - \\
\hline Absent & 20 & 18 & - & - & - & - \\
\hline
\end{tabular}

MRI - magnetic resonance imaging, OR - odds ratio, Cl - confidence interval, MPD - main pancreatic duct, ADC - apparent diffusion coefficient, LSR - lesion-to-spine ratio, EUS - endoscopic ultrasonography, IPMN - intraductal papillary mucinous neoplasm, ROI - region of interest

Prior to statistical analysis, an f-test was applied to age, ADC values, area of ROI for ADC, LSR and the area of ROl for LSR to test for a normal distribution. All variables showed no significant difference except for LSR at the 5\% significance level. Therefore, the Wilcoxon rank sum test was used for LSR.

a slice of the lumbar enlargement because of its larger signal intensity compared with those of other regions of the spine, which facilitated location of the ROI.

LSR was calculated according to the following formula:

LSR = mean signal intensity of lesion on DWI/maximum signal intensity of spinal cord on DWI.

\section{Blinded evaluation}

For comparison of the diagnostic performance of MRI with EUS and MRI alone in distinguishing malignant from benign IPMNs, three radiologists $(15,8$, and 7 years of experience in interpreting MR images, respectively) who were blinded to clinical and histopathological information, reviewed the image sets, i.e. preoperative MRI alone and MRI with EUS. Each image set was reviewed in the same order (first, MRI only; second, MRI with EUS) by three blinded readers to assess the following: MPD size (>10 mm or 5-9 mm or not); cyst size (> $30 \mathrm{~mm}$ or not); wall and/ or septal thickness of cyst ( $>3 \mathrm{~mm}$ or not); mural nodule height and/or width (> $10 \mathrm{~mm}$ or 5-9 $\mathrm{mm}$ or not); abrupt change in the MPD calibre with or without distal pancreatic atrophy (present or absent); lymphadenopathy (present or absent); areas of restricted diffusion (present or absent); and signal alteration of pancreatic parenchyma and/or peripancreatic fat tissue (present or absent).

Restricted diffusion was defined by DWI findings that satisfied the following two conditions: (i) for qualitative assessment, when both high-signal intensity regions compared with normal pancreas parenchyma on the high $\mathrm{b}$ value DWIs ( $\mathrm{b}$-value $=800 \mathrm{~s} / \mathrm{mm}^{2}$ ) and low signal intensity regions on the ADC map with high LSR was detected; (ii) for quantitative assessment, the optimal cut-off LSR value determined on ROC curve analysis was used to determine the presence or absence of restricted diffusion. To evaluate the diagnostic values of other MR findings suggestive of IPMC with an invasive component, the presence of signal alteration in the pancreatic parenchyma or peripancreatic fat tissue was also evaluated. The readers were finally asked to evaluate the possibility of malignancy using the following previously reported scoring criteria $[15,16]$ : score 1, no WFs or HRS; score 2, one WF with no HRS; score 3, more than two WFs with no HRS; score 4, one HRS; and score 5, more than two HRS. Radiological WFs included cyst size, wall and/or septal thickness of cyst; mural nodule without a restricted diffusion, abrupt change in the MPD calibre with distal pancreatic atrophy, and lymphadenopathy. Radiological HRSs included cystic lesion of the pancreatic head with dilatation of the biliary tract, mural nodule with a restricted diffusion, and MPD dilatation $>10 \mathrm{~mm}$. A mural nodule with a restricted diffusion was defined as equivalent to an enhanced mural nodule. In the blinded reading of MRI with EUS, the written results of EUS were provided to each reader after the first reading of MRI alone. The EUS findings included findings associated with international consensus guidelines 2012 for the management of IPMN and MCN of the pancreas [10]. No blood flow information of the lesion was included. The MRI findings that changed according to the results of the EUS findings were recorded. Subsequently, each reader was asked to report a final decision by using the EUS results. At this point, the readers were asked to prioritise the EUS findings, if required. 


\section{Histopathological analysis}

In the present study, low- and intermediate-grade dysplasia were defined as IPMN adenomas (IPMAs), whereas high-grade dysplasia and IPMN with carcinoma were defined as IPMCs, as previously described [15].

\section{Statistical analysis}

All statistical analyses were performed using EkuseruToukei 2015 (SSRI, Tokyo, Japan) and R (The R Project for Statistical Computing, www.r-project.org, version 3.3.0) software.

For preliminary univariate analyses, a two-tailed paired $t$-test was used to compare the mean age, ADC, the area of ROI for ADC, and the area of ROI for LSR between the benign and malignant lesions. For LSR, the Wilcoxon rank sum test was used. Other findings were analysed using the Fisher exact test in the univariate analyses. A $p$ value $<0.05$ was considered statistically significant. Multivariate logistic regression analyses were conducted with backward selection using variables that showed a significant association on univariate analyses, and by referring to significant findings reported elsewhere $[10,17,18]$.

Based on the blinded scores, an ROC curve was fit to each reader's confidence rating using maximum-likelihood estimation to determine observer performance and cut-off values. Observer performance was individually calculated based on the area under the ROC curve (AUC). For assessment of the diagnostic performance of MRI with and without EUS and the prediction of invasive IPMC in the blinded evaluation, the sensitivity, specificity, positive-predictive value, and negative-predictive value were also determined for each reader. The assessment of each reader's findings with and without information of EUS findings was performed using the McNemar test. To determine the agreement rate of scoring among the three blinded readers, weighted $\kappa$ statistics was used to measure the degree of agreement respectively. A value of $\leq 0.20$ was considered as slight agreement, $0.21-0.40$ as fair agreement, 0.41-0.60 as moderate agreement, 0.610.80 as substantial agreement, and $\geq 0.81$ as almost perfect agreement.

\section{Ethical approval}

This study was approved by the institutional review board of The Jikei University School of Medicine (approval number 28-160).

\section{Results}

The study included 38 patients with 20 histopathologically confirmed IPMC lesions and 18 IPMA lesions. The IPMC lesions included two with high-grade dysplasia, six with non-invasive carcinoma, and 12 with invasive carcinoma.

\section{Evaluation of imaging findings for preliminary analyses}

Findings of patients with IPMCs and IPMAs are shown in Table 1. On univariate analyses of MRI findings, statistically significant findings for the discrimination of malignant lesions from benign lesions were MPD size ( $\geq 5 \mathrm{~mm}$ ), abrupt change in the MPD calibre with distal pancreatic atrophy, ADC value, and LSR (all $p<0.001$ ). On multivariate analyses of MRI findings, both abrupt change in the MPD calibre with distal pancreatic atrophy and LSR demonstrated a statistically significant difference (odds ratio [OR]: 34.5; 95\% confidence interval [CI]: 1.85-644; $p=0.018$; OR: 16.3 ; 95\% CI: 1.45-184; $p=0.02$, respectively).

On univariate analyses of EUS findings, statistically significant findings for the discrimination of malignant lesions from benign lesions were MPD size ( $\geq 5 \mathrm{~mm}$ ), Wall and/or septal thickness of cyst, Mural nodule height and/or width, and abrupt change in the MPD calibre with distal pancreatic atrophy (all $p<0.05)$. On multivariate analyses of EUS findings, only MPD size ( $\geq 5 \mathrm{~mm}$ ) exhibited a statistically significant difference (OR: 8.15; 95\% CI: 1.04-63.9; $p=0.045$ ).

\section{Measurement of apparent diffusion coefficient and lesion-to-spinal cord ratio using diffusion-weighted images}

ADC values and LSR in DW images are shown in Figure 2. The cut-off ADC value for the diagnosis of IPMC was $\leq 2.12 \times 10^{-3} \mathrm{~mm}^{2} / \mathrm{s}$. The cut-off LSR value for the diagnosis of IPMC was $\geq 0.18$. The mean ADC for IPMC was significantly lower than that for IPMA $(p<0.001)$. On the other hand, the mean LSR for IPMC was significantly higher than that for IPMA $(p<0.001)$.

\section{Blinded evaluation}

The diagnostic performance of MRI with and without EUS in distinguishing malignant IPMNs from benign IPMNs using blinded evaluation is shown in Table 2 . The three blinded readers showed no significant difference in AUCs for MRI alone and MRI with EUS (reader 1: 95\% CI: $-0.083-0.027 ; p=0.32$; reader 2 : $95 \% \mathrm{CI}:-0.034-$ 0.012 ; $p=0.34$; reader $3: 95 \% \mathrm{CI}:-0.121-0.025 ; p=0.2$ ). No significant difference was observed in the sensitivity, specificity, and positive- and negative-predictive values of MRI alone and MRI with EUS. There were differences between the three readers with respect to findings of mural nodule, wall and/or septal thickness of cyst, and MPD size (Figure 3). However, the difference was not statistically significant (mural nodule: $p=0.25$ to $>0.99$; wall and/ or septal thickness of cyst: $p>0.99$; MPD size: $p=0.25$ ). The prediction of invasive IPMC is shown in Table 3. The three readers generally showed lower sensitivity and higher specificity irrespective of the presence or ab- 

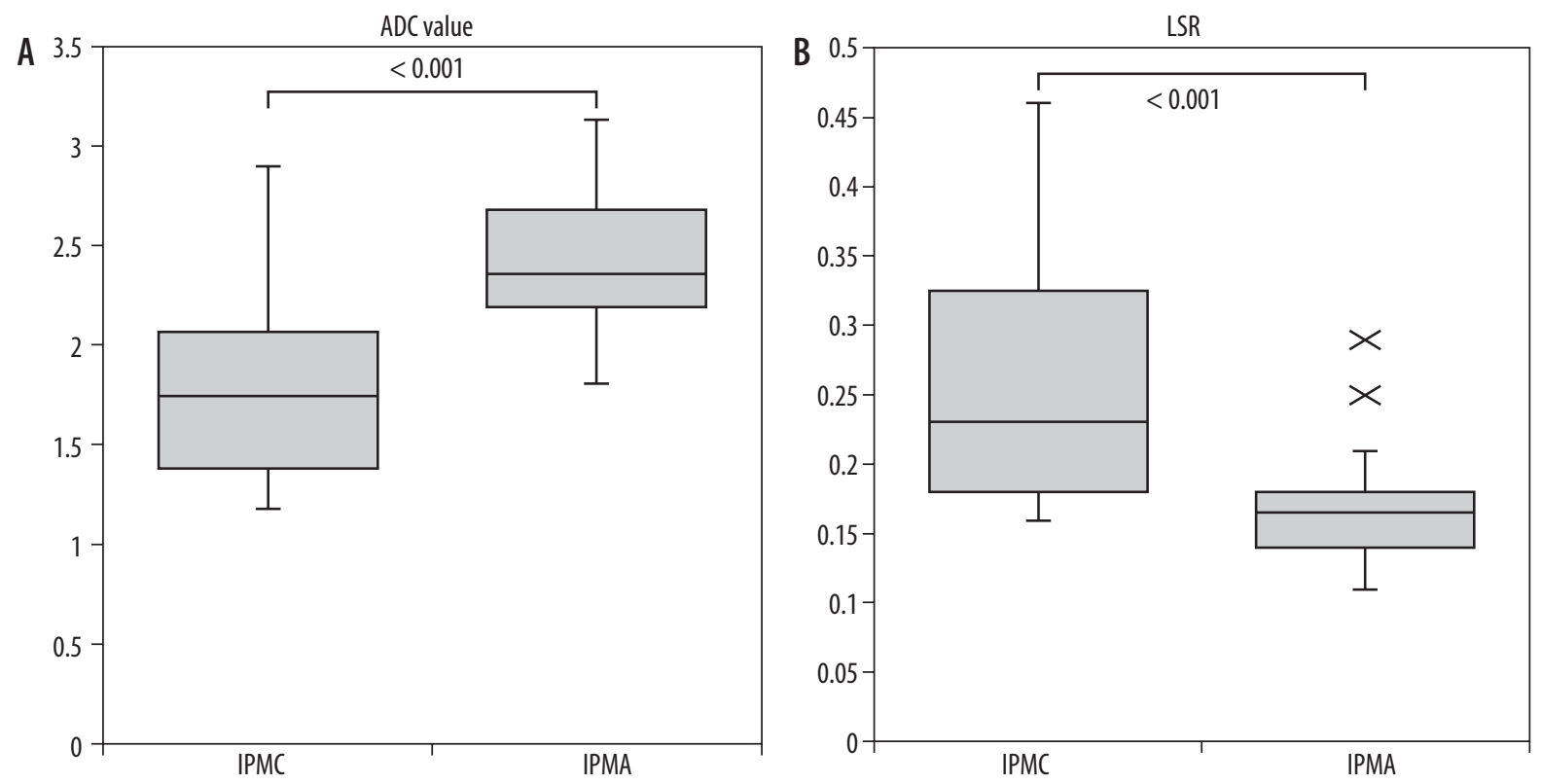

DWI- diffusion-weighted imaging

Figure 2. Apparent diffusion coefficient (ADC) and lesion-to-spinal cord ratio on diffusion-weighted imaging. Mean ADC for intraductal papillary mucinous adenocarcinoma (IPMC) was significantly lower than that for intraductal papillary mucinous adenoma (IPMA). The mean lesion-to-spinal cord ratio (LSR) for IPMC was significantly higher than that for IPMA

Table 2. Diagnostic performance of magnetic resonance imaging (MRI) with and without use of endoscopic ultrasonography (EUS), in distinguishing malignant from benign intraductal papillary mucinous neoplasms on blinded evaluation

\begin{tabular}{|c|c|c|c|c|c|c|c|c|c|c|c|}
\hline & \multicolumn{4}{|c|}{ AUC } & \multicolumn{2}{c|}{ Sensitivity } & \multicolumn{2}{c|}{ Specifity } & \multicolumn{2}{|c|}{ PPV } & \multicolumn{2}{|c|}{ NPV } \\
\cline { 2 - 12 } & MRI alone & MRI with EUS & $p$-value & $\begin{array}{c}\text { MRI } \\
\text { alone }\end{array}$ & $\begin{array}{c}\text { MRI } \\
\text { with } \\
\text { EUS }\end{array}$ & $\begin{array}{c}\text { MRI } \\
\text { alone }\end{array}$ & $\begin{array}{c}\text { MRI } \\
\text { with } \\
\text { EUS }\end{array}$ & $\begin{array}{c}\text { MRI } \\
\text { alone }\end{array}$ & $\begin{array}{c}\text { MRI } \\
\text { with } \\
\text { EUS }\end{array}$ & $\begin{array}{c}\text { MRI } \\
\text { alone }\end{array}$ & $\begin{array}{c}\text { MRI } \\
\text { with } \\
\text { EUS }\end{array}$ \\
\hline Reader1 & $\begin{array}{c}0.75 \\
(0.598-0.908)\end{array}$ & $\begin{array}{c}0.78 \\
(0.632-0.929)\end{array}$ & $\begin{array}{c}0.32 \\
(-0.083-0.027)\end{array}$ & 72.2 & 72.2 & 75.0 & 80.0 & 72.2 & 76.5 & 75.0 & 76.2 \\
\hline Reader2 & $\begin{array}{c}0.71 \\
(0.08-0.554)\end{array}$ & $\begin{array}{c}0.72 \\
(0.57-0.87)\end{array}$ & $\begin{array}{c}0.34 \\
(-0.034-0.012)\end{array}$ & 55.6 & 55.6 & 80.0 & 80.0 & 71.4 & 71.4 & 66.7 & 66.7 \\
\hline Reader3 & $\begin{array}{c}0.63 \\
(0.422-0.828)\end{array}$ & $\begin{array}{c}0.67 \\
(0.473-0.873)\end{array}$ & $\begin{array}{c}0.2 \\
(-0.121-0.025)\end{array}$ & 75 & 75 & 69.2 & 69.2 & 52.9 & 52.9 & 85.7 & 85.7 \\
\hline
\end{tabular}

Sensitivity, specificity, PPV, and NPV values are expressed as percentages.

Data in parentheses are $95 \%$ confidence intervals.

PPV - positive-predictive value, NPV - negative-predictive value, AUC - area under the ROC curve

sence of EUS information. The interobserver agreement between the three blinded readers is shown in Table 4 . A moderate to substantial agreement was observed for the prediction of malignant IPMNs $(\kappa=0.57-0.79)$. MRI with EUS generally demonstrated higher interobserver agreement than MRI alone for the prediction of malignant IPMNs. The same tendency was not observed for the prediction of invasive IPMCs $(\kappa=0.43-0.68)$ (Figure 4).

\section{Discussion}

In the present retrospective study, we compared the diagnostic performance of non-enhanced MRI with non-enhanced EUS to that of non-enhanced MRI alone for predicting the malignant potential of IPMNs, by performing a blinded evaluation. Combined use of non-enhanced MRI and EUS improved the ability to diagnose malignant IPMNs; however, this improvement was not statistically significant. The effectiveness of measuring ADC values and LSR for predicting malignant potential has been assessed in previous reports $[5,8,9,19,20]$. Our finding that the ADC values of high-grade/invasive IPMN lesions were significantly lower than those of low-grade IPMN lesions is consistent with the findings of earlier studies $[5,9]$. The use of LSR in the present study also helped distinguish malignant from benign IPMNs; similar findings have been reported elsewhere in the context of lung cancer and gall bladder $[19,20]$. It is relatively convenient to calculate LSR on a PACS monitor independent of the MRI device. However, further studies are required to clarify the 

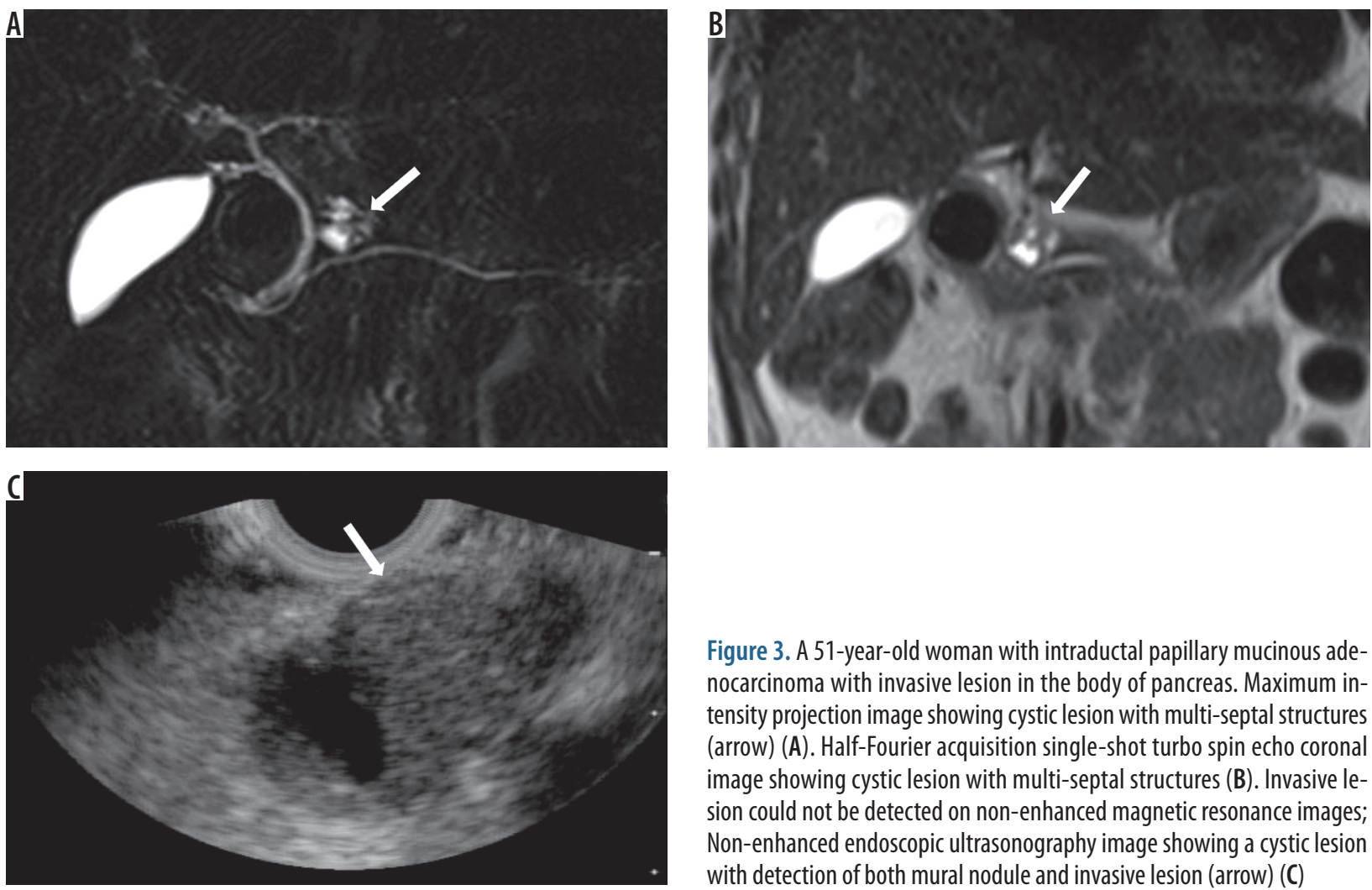

Figure 3. A 51-year-old woman with intraductal papillary mucinous adenocarcinoma with invasive lesion in the body of pancreas. Maximum intensity projection image showing cystic lesion with multi-septal structures (arrow) (A). Half-Fourier acquisition single-shot turbo spin echo coronal image showing cystic lesion with multi-septal structures (B). Invasive lesion could not be detected on non-enhanced magnetic resonance images; Non-enhanced endoscopic ultrasonography image showing a cystic lesion with detection of both mural nodule and invasive lesion (arrow) (C)

Table 3. Prediction of invasive intraductal papillary mucinous adenocarcinoma

\begin{tabular}{|l|c|c|c|c|c|c|c|c|}
\multirow{2}{*}{} & \multicolumn{2}{|c|}{ Sensitivity } & \multicolumn{2}{c|}{ Specificity } & \multicolumn{2}{c|}{ PPV } & \multicolumn{2}{c|}{ NPV } \\
\cline { 2 - 10 } & MRI alone & MRI with EUS & MRI alone & MRI with EUS & MRI alone & MRI with EUS & MRI alone & MRI with EUS \\
\hline Reader 1 & 50.0 & 58.3 & 92.0 & 92.3 & 75.0 & 77.8 & 80.0 & 82.8 \\
\hline Reader 2 & 58.3 & 66.7 & 96.2 & 96.2 & 87.5 & 88.9 & 83.3 & 86.2 \\
\hline Reader 3 & 25.0 & 25.0 & 100 & 100 & 100 & 100 & 74.3 & 74.3 \\
\hline
\end{tabular}

All parameters expressed as percentages.

PPV - positive-predictive value, NPV - negative-predictive value, MRI - magnetic resonance imaging, EUS - endoscopic ultrasonography

Table 4. Weighted $\kappa$ values of interobserver agreement between the three blinded readers

\begin{tabular}{|l|c|c|c|c|}
\hline \multirow{2}{*}{} & \multicolumn{2}{|c|}{ Prediction of malignant IPMN } & \multicolumn{2}{c|}{ Prediction of invasive IPMC } \\
\cline { 2 - 5 } & MRI alone & MRI with EUS & MRI alone & MRI with EUS \\
\hline Reader 1 and 2 & $0.67(0.498-0.842)$ & $0.67(0.473-0.861)$ & $0.68(0.395-0.971)$ & $0.62(0.321-0.922)$ \\
\hline Reader 2 and 3 & $0.57(0.33-0.818)$ & $0.65(0.433-0.866)$ & $0.48(0.127-0.846)$ & $0.43(0.09-0.776)$ \\
\hline Reader 1 and 3 & $0.72(0.565-0.877)$ & $0.79(0.648-0.929)$ & $0.49(0.127-0.846)$ & $0.49(0.127-0.846)$ \\
\hline
\end{tabular}

K-values with 95\% Confidence Intervals are presented.

IPMN - intraductal papillary mucinous neoplasms, IPMC - intraductal papillary mucinous adenocarcinoma, MRI - magnetic resonance imaging, EUS - endoscopic ultrasonography

utility of LSR vis-à-vis that of ADC. Our study suggested a tendency for lower sensitivity and higher specificity for predicting malignant potential of IPMNs and invasive IPMCs on blinded evaluation. This suggests that evaluation of a score of 2 and 3 that includes one WF or more than two WFs with no HRS could impact the sensitivity. It has been previously shown that radiological WFs are not cumulative in risk prediction because there is no stepwise increase in rates of malignant or invasive IPMN with an increase in the number of WFs [21].

In the blinded evaluation, the addition of EUS findings changed the interpretation of mural nodule, wall and/ 

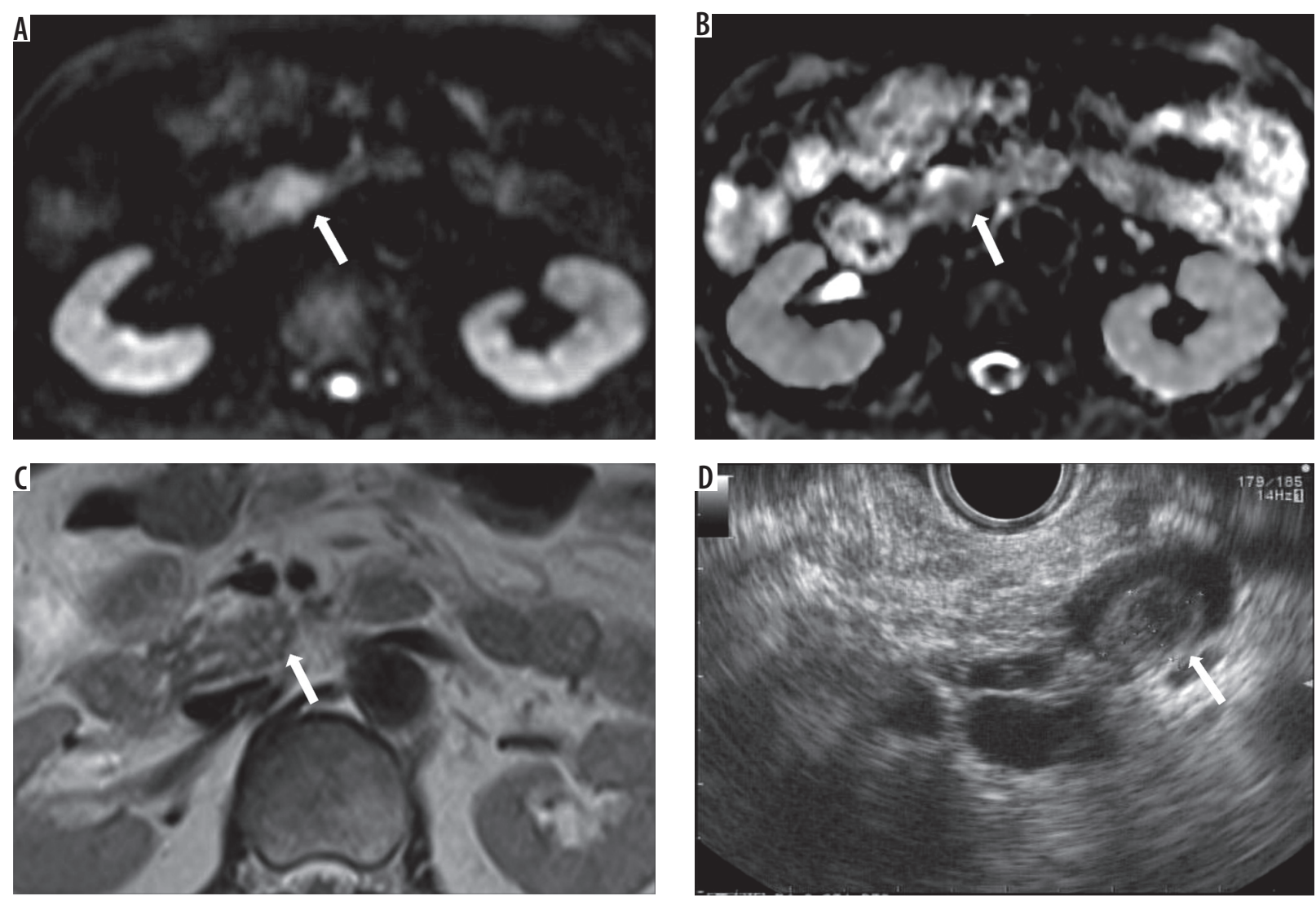

Figure 4. An 82-year-old man with intraductal papillary mucinous adenocarcinoma with invasive lesion in the head of pancreas. Multilocular cystic mass with mural nodules was detected on non-enhanced magnetic resonance imaging. An invasive lesion with restricted diffusion was detected on diffusion-weighted image (arrow) (A). Restricted diffusion was identified as a low-signal lesion on apparent diffusion coefficient map (arrow) (B). The uncinate process of pancreas showed loss of normal morphology due to invasive lesion of the intraductal papillary mucinous adenocarcinoma on T2-weighted image (arrow) (C). A multilocular cystic mass with mural nodules (arrow) was detected by endoscopic ultrasonography, which failed to identify the invasive lesion (D)

or septal thickness of cyst, and MPD size on MRI by the three readers. However, the change was statistically insignificant. Non-enhanced MRI with DWI has only a limited value for diagnosis of high-grade dysplasia and stage $\mathrm{T} 1$ carcinoma [7]. The low prevalence of a solid proliferative portion in both non-invasive IPMC and benign IPMN was reported to underlie the significant overlap of ADC values between these two lesions [5]. Furthermore, depending on the viscosity of the contents of cystic lesions and/or mucus plugs, the ADC values and LSR of lesions can show various values. From this perspective, assessment of vascularity for solid proliferative portion may be necessary. On the basis of the FG criteria that accord weight to an enhancing mural nodule for the purpose of determining malignancy [10], our findings suggest that blood flow information is necessary for the interpretation of WFs, especially for mural nodules and wall and/or septal thickness of cyst.

Diagnostic accuracy study demonstrated that the FG criteria, which was selected in our study, has higher sensitivity in detecting high-grade dysplasia and cancer compared to the two guidelines created by the 2015 American Gastroenterological Association and the American College of Radiology [22].
As stated in the FG criteria, if we were able to add the EUS findings with blood flow data of the lesion, when evaluating the non-enhanced MRI findings, the sensitivity of our results could have been improved, especially in cases that scored 2 or 3 on blinded evaluation.

Contrast-enhanced harmonic EUS with time-intensity curve analysis was shown to be useful for quantitative evaluation of blood flow in the IPMN microvasculature, and for differentiating between high-grade dysplasia/invasive carcinoma and low- and intermediate-grade dysplasia [23]. In clinical practice, although a non-enhanced EUS as a screening modality can detect pancreatic intraepithelial neoplasia lesions and small invasive carcinomas associated with IPMN $[24,25]$ in the case of lesions scored as 2 or 3 , it is reasonable to recommend enhanced EUS in addition to non-enhanced MRI. Presently, there is no consensus on the use of dynamic contrast enhanced MRI for distinguishing malignant from benign IPMNs. Further studies are required to assess the relative value of enhanced MRI and enhanced EUS.

Several limitations of the present study should be acknowledged. The retrospective study design and relatively small sample size are key limitations that limit the statistical power of the analysis and may have introduced an element of selection bias. In particular, changes in FG 
(2012) might have influenced the patient inclusion criteria. Secondly, in some cases it was difficult to distinguish mural nodule from mucous plug or normal pancreatic parenchyma in the preliminary analyses. This may have influenced the interpretation of MRI findings in the blinded evaluation. Thirdly, the EUS criteria for IPMNs were mainly the MPD and internal characteristics of the cystic lesion; there was inadequate emphasis on the presence or absence of findings suggestive of IPMC with an invasive component. This could have had an impact on the two readers with lower $\kappa$ values for MRI with EUS than for MRI alone, in the prediction of invasive IPMC.

In conclusion, the clinical utility of non-enhanced EUS may be attributive in evaluating IPMN that has al- ready been evaluated by non-enhanced MRI. The results of our analysis suggest that the additional use of enhanced EUS for patients with one WF or more than two WFs and no HRS on non-enhanced MRI may be useful.

\section{Acknowledgements}

The authors would like to thank Soumei Nozu (Siemens Japan K. K., Customer Service Division) for providing information about detailed MR parameters.

\section{Conflict of interest}

The authors report no conflict of interest.

\section{References}

1. Laffan TA, Horton KM, Klein AP, et al. Prevalence of unsuspected pancreatic cysts on MDCT. AJR Am J Roentgenol 2008; 191: 802-807.

2. Hruban RH, Takaori K, Klimstra DS, et al. An illustrated consensus on the classification of pancreatic intraepithelial neoplasia and intraductal papillary mucinous neoplasms. Am J Surg Pathol 2004; 28: 977-987.

3. Matthaei H, Schulick RD, Hruban RH, Maitra A. Cystic precursors to invasive pancreatic cancer. Nat Rev Gastroenterol Hepatol 2011; 8: $141-150$.

4. Bassi C, Sarr MG, Lillemoe KD, Reber HA. Natural history of intraductal papillary mucinous neoplasms (IPMN): Current evidence and implications for management. J Gastrointest Surg 2008; 12: 645-650.

5. Kang KM, Lee JM, Shin CI, et al. Added value of diffusion-weighted imaging to MR cholangiopancreatography with unenhanced MR imaging for predicting malignancy or invasiveness of intraductal papillary mucinous neoplasm of the pancreas. J Magn Reson Imaging 2013; 38: 555-563.

6. Ogawa T, Horaguchi J, Fujita N, et al. Diffusion-weighted magnetic resonance imaging for evaluating the histological degree of malignancy in patients with intraductal papillary mucinous neoplasm. J Hepatobiliary Pancreat Sci 2014; 21: 801-808.

7. Jang KM, Kim SH, Min JH, et al. Value of diffusion-weighted MRI for differentiating malignant from benign intraductal papillary mucinous neoplasms of the pancreas. AJR Am J Roentgenol 2014; 203: 992-1000.

8. Kartalis N, Lindholm TL, Aspelin P, et al. Diffusion-weighted magnetic resonance imaging of pancreas tumours. Eur Radiol 2009; 19: 1981-1990.

9. Sandrasegaran K, Akisik FM, Patel AA, et al. Diffusion-weighted imaging in characterization of cystic pancreatic lesions. Clin Radiol 2011; 66: 808-814.

10. Tanaka M, Fernández-del Castillo $\mathrm{C}$, Adsay V, et al. International consensus guidelines 2012 for the management of IPMN and MCN of the pancreas. Pancreatology 2012; 12: 183-197.

11. Ohno E, Itoh A, Kawashima H, et al. Malignant transformation of branch duct-type intraductal papillary mucinous neoplasms of the pancreas based on contrast-enhanced endoscopic ultrasonography morphological changes: Focus on malignant transformation of in-

traductal papillary mucinous neoplasm itself. Pancreas 2012; 41: 855-862.

12. Kobayashi N, Sugimori K, Shimamura T, et al. Endoscopic ultrasonographic findings predict the risk of carcinoma in branch duct intraductal papillary mucinous neoplasms of the pancreas. Pancreatology 2012; 12: 141-145.

13. Inui K, Kida M, Fujita N, et al. Standard imaging techniques in the pancreatobiliary region using radial scanning endoscopic ultrasonography. Dig Endosc 2004; 16: S118-S133.

14. EUS-FNA Standardization Committee, Yamao K, Irisawa A, et al. Standard imaging techniques of endoscopic ultrasound-guided fine-needle aspiration using curved linear array echoendoscope. Dig Endosc 2007; 19: S180-S205.

15. Kim SH, Lee JM, Lee ES, et al. Intraductal papillary mucinous neoplasms of the pancreas: evaluation of malignant potential and surgical resectability by using MR imaging with MR cholangiography. Radiology 2015; 274: 723-733.

16. Kang HJ, Lee JM, Joo I, et al. Assessment of malignant potential in intraductal papillary mucinous neoplasms of the pancreas: Comparison between multidetector CT and MR imaging with MR cholangiopancreatography. Radiology 2016; 279: 128-139.

17. Kawada N, Uehara H, Nagata S, et al. Mural nodule of $10 \mathrm{~mm}$ or larger as predictor of malignancy for intraductal papillary mucinous neoplasm of the pancreas: Pathological and radiological evaluations. Pancreatology 2016; 16: 441-448.

18. Ridtitid W, DeWitt JM, Schmidt CM, et al. Management of branchduct intraductal papillary mucinous neoplasms: a large single-center study to assess predictors of malignancy and long-term outcomes. Gastrointest Endosc 2016; 84: 436-445.

19. Uto T, Takehara Y, Nakamura Y, et al. Higher sensitivity and specificity for diffusion-weighted imaging of malignant lung lesions without apparent diffusion coefficient quantification. Radiology 2009; 252: 247-254

20. Kitazume Y, Taura S, Nakaminato S, et al. Diffusion-weighted magnetic resonance imaging to differentiate malignant from benign gallbladder disorders. Eur J Radiol 2016; 85: 864-873.

21. Roch AM, Ceppa EP, DeWitt JM, et al. International Consensus Guidelines parameters for the prediction of malignancy in intra- 
ductal papillary mucinous neoplasm are not properly weighted and are not cumulative. HPB (Oxford) 2014; 16: 929-935.

22. Xu MM, Yin S, Siddiqui AA, et al. Comparison of the diagnostic accuracy of three current guidelines for the evaluation of asymptomatic pancreatic cystic neoplasms. Medicine (Baltimore) 2017; 96: e7900.

23. Yamamoto N, Kato H, Tomoda T, et al. Contrast-enhanced harmonic endoscopic ultrasonography with time-intensity curve analysis for intraductal papillary mucinous neoplasms of the pancreas. Endoscopy 2016; 48: 26-34.
24. Maire F, Couvelard A, Palazzo L, et al. Pancreatic intraepithelial neoplasia in patients with intraductal papillary mucinous neoplasms: the interest of endoscopic ultrasonography. Pancreas 2013; 42: 12621266.

25. Kamata K, Kitano M, Kudo M, et al. Value of EUS in early detection of pancreatic ductal adenocarcinomas in patients with intraductal papillary mucinous neoplasms. Endoscopy 2014; 46: 22-29. 